Please do not remove this page

RMIT

UNIVERSITY

\title{
Stripping very low frequency communication signals with minimum shift keying encoding from streamed time-domain electromagnetic data
}

Macnae, James

https://researchrepository.rmit.edu.au/esploro/outputs/9921862745001341/filesAndLinks?institution=61RMIT_INST\&index=null

Macnae, J. (2015). Stripping very low frequency communication signals with minimum shift keying encoding from streamed time-domain electromagnetic data. Geophysics, 80(6), E343-E353. https://doi.org/10.1190/GEO2015-0304.1

Document Version: Published Version

Published Version: https://doi.org/10.1190/GEO2015-0304.1

Repository homepage: https://researchrepository.rmit.edu.au (c) 2015 Society of Exploration Geophysicists. All rights reserved.

Downloaded On 2023/04/26 11:52:19 +1000 
Thank you for downloading this document from the RMIT Research Repository.

The RMIT Research Repository is an open access database showcasing the research outputs of RMIT University researchers.

RMIT Research Repository: http://researchbank.rmit.edu.au/

\section{Citation:}

Macnae, J 2015, 'Stripping very low frequency communication signals with minimum shift keying encoding from streamed time-domain electromagnetic data', Geophysics, vol. 80, no. 6, pp. E343-E353.

See this record in the RMIT Research Repository at:

https://researchbank.rmit.edu.au/view/rmit:35009

Version: Published Version

Copyright Statement: (c) 2015 Society of Exploration Geophysicists.

Link to Published Version:

http://dx.doi.org/10.1190/GEO2015-0304.1 


\title{
Stripping very low frequency communication signals with minimum shift keying encoding from streamed time-domain electromagnetic data
}

\author{
James Macnae ${ }^{1}$
}

\begin{abstract}
The objective of this research is to eliminate very low frequency (VLF) communication signals with frequencies in the range of $15-25 \mathrm{kHz}$ from streamed time-domain electromagnetic data. The frequency-domain approach of notch filtering or bandwidth limitation is unsatisfactory when early delay-time data are required in a time-domain system. United States military VLF transmitters presently communicate 200 bits/s using minimum shift keying modulation, and it is possible to derive the encrypted bitstream from the data sampled at typical geophysical streaming rates. The method involves convolving the data with waveforms of frequency different by one-quarter of the bit rate above and below the carrier frequency and using
\end{abstract}

the difference between the convolutions to predict the transmitted bits. The transmitted signal is then exactly recreated from the decoded bits, and the predicted signal at the receiver is subtracted from the data stream. It is possible to predict bit rates and encoding methods from other military transmitters through data analysis and again subtract the predicted signals from the streamed data. This procedure reduces the variance of data, implying that unwanted VLF signals have been reduced by a factor of three to nine in stations thousands of kilometers from a VLF transmitter. Much larger signal/noise improvements are predicted for stations within a few hundred kilometers of the VLF source. Lower degrees of improvement are noted from Indian and Chinese transmitters, which appear to have different encoding and modulation methods.

\section{INTRODUCTION}

The use of high frequencies $>10 \mathrm{kHz}$ or early time samples $<0.1 \mathrm{~ms}$ in electromagnetic geophysics has long been known to be appropriate for shallow investigations in electrically resistive areas (Everett, 2013). A recent advance in ground electromagnetic receivers that facilitates collection of very early delay time data, airborne electromagnetic (AEM) systems, and audio-frequency magnetotellurics (AMT) equipment has been the collection and storage of the streamed data at high sample rates (Lane et al., 1998; Schamper et al., 2014; Hennessy and Macnae, 2015).

In these streamed data, with sampling frequency between 50 and $192 \mathrm{kHz}$, very low frequency (VLF) transmissions for submarine communications are a major source of wanted or unwanted signal in the $15-$ to $25-\mathrm{kHz}$ band. Several of these VLF transmitters consume power in the several hundred to a thousand kilowatt range, and their signals can usually be detected 10,000 km from the source (Barr et al., 2000) or a few tens of meters under water (Waheed-uz-Zaman and Yousufzai, 2010). Several geophysical instruments can tune to individual or multiple VLF stations and use amplitude and phase information in different directional components or electric/magnetic field rations to derive earth conductivity or target location information (Pedersen and Oskooi, 2004). Information can be extracted regarding conductivity to depths of up to $200 \mathrm{~m}$ in crystalline crustal areas, such as Sweden (Oskooi and Pedersen, 2006).

I will use the term encryption to mean the process of transforming a text message into a sequence of binary numbers that only make sense to the intended recipient of the message. I will use the term encoding in a broadcast communications sense to represent the conversion of this binary sequence into a form suitable for transmission. The term modulation is also commonly used in the communication literature in the same sense as "encoding." The term decoding, then, will logically be the conversion of a received transmission into its original binary sequence.

VLF military transmitters built to transmit encrypted messages are major installations easily visible on Google Earth, but their frequencies and binary encoding methods do occasionally change. 
To my knowledge, there is no definitive and up-to-date public list of active transmitters and their encoding schemes. A Google search will locate many incomplete and often outdated lists of VLF transmitter latitude, longitude, and frequency. Some lists usefully contain bit or baud rates as well as encoding and modulation methods, but again the information on frequency and encoding is often outdated.

At any survey site on earth, the transmissions of many VLF sources can usually be detected because signals at these frequencies propagate well in the earth-ionosphere waveguide (Thomson et al., 2011). Generally, unless distorted by severe topography, regional conductors or refraction by the day-night ionosphere boundary, the horizontal or the primary magnetic field is perpendicular to the great circle path from the transmitter (Barr et al., 2000). The direction to the source can be estimated using the amplitudes of east-west and north-south sensors, or through the radio direction finding method of rotating a horizontal sensor to determine maximum and/or null coupling (Pedersen, 1998; Oskooi and Pedersen, 2005). The closest active high-power VLF transmitters in Eastern Australia are shown in Figure 1, together with great circle paths to an RMIT field test site (labeled as site 1) located in the north of Melbourne, Victoria. Highpower United States Navy transmitters are NWC in Western Australia and NPM in Hawaii, and the United States transmitters are reported to have steady power output except during weekly maintenance (Thomson et al., 2011). Even with steady power output of a VLF transmitter, the signal strength received at a distant station can vary significantly due to multipath interference and ionospheric height changes caused by the solar wind variations particularly the differences across the day-night boundary (Vallée et al., 1992a, 1992b; Thomson et al., 2011; Samanes et al., 2015).

From a controlled-source perspective, VLF signals have been regarded as noise (Efferso et al., 1999). In frequency-domain systems, acquisition tends to be narrowband at the frequency of transmission,

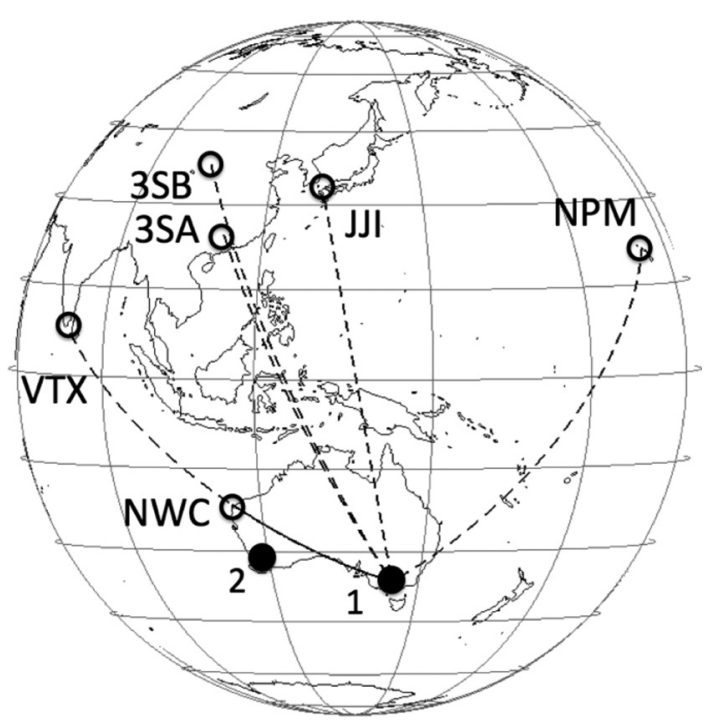

Figure 1. Call signs of the closest high-power VLF transmitters in Eastern Australia. Great circle paths from these transmitters to test site 1, near Heathcote, Victoria, are shown. The NWC transmitter in Western Australia lies on the same great circle path as the more distant VTX transmitter near the tip of the Indian subcontinent. Test site 2 is $1200 \mathrm{~km}$ south of the NWC transmitter and in this case, VLF signal rejection is trivial. Swept frequency systems are possible, but they are not in common use in geophysics.

Little if anything has been published in the geophysical literature on stripping the effects of VLF communication signals from controlled-source time-domain data. One author is Allard (2007), who states: "VLF noise, like the sferics noise, affects mainly the horizontal components. Since VLF are radio waves in the $15-25 \mathrm{kHz}$ frequency range, their effect is eliminated by a notch filter or significantly reduced by averaging during the stacking process."

Some contractors successfully cut the high-frequency response of their receiver systems to eliminate VLF. Using a $15-\mathrm{kHz}$, secondorder Butterworth band-limit filter (Efferso et al., 1999; Christiansen et al., 2011; Macnae, 2015) causes the instantaneous step to appear as a smoothed transition with an average $20 \mu$ s delay but with effects lasting to approximately $100 \mu \mathrm{s}$, which is illustrated in Figure 2. I am aware of unsuccessful attempts by two different airborne contractors and several ground EM system developers to minimize the effects of VLF by notch filtering. Figure $2 \mathrm{c}$ shows one unavoidable and severe side effect of narrowband frequencydomain filtering, illustrated here with a step-off waveform. Using a 200-Hz-wide, $19.8-\mathrm{kHz}$ notch filter, the step change in amplitude induces ringing in the off-time response with a gradual envelope decay as shown in Figure 2c. This ringing is in fact excited by any abrupt transition in the electromagnetic signal received by a sensor. Some further aspects of notch filters are discussed in Appendix A. I am therefore suspicious of contractor claims to successful notch filtering as reported by Allard (2007).

Neither band limiting nor notch filtering to minimize VLF signals can be applied to the data stream if quantitative time-domain data are required in the microsecond or tens of microseconds range. There are some finite impulse response notch filters that shorten the duration of ringing as shown in Figure 2c, but they do not solve the problem at very early delay times. As Allard (2007) suggests, time averaging helps to reduce VLF signal noise in AEM data, but often this averaging is insufficient.

In the geophysical literature, the assumption that VLF transmissions occur at a constant frequency is ubiquitous (e.g., McNeill and Labson, 1991; Pedersen and Oskooi, 2004) and has proven

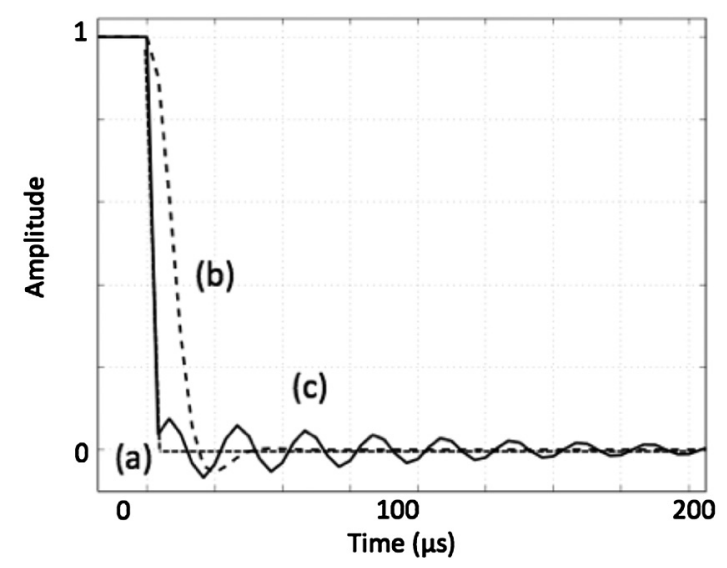

Figure 2. Using (a) a step-off waveform at time zero, there is a delaying and smoothing effect of (b) a $15-\mathrm{kHz}$ band-limit filter on the response. (c) This profile shows the oscillatory effect of a $19.8-\mathrm{kHz}$, 500-Hz-wide notch filter applied to the waveform. 
to generate interpretable and useful results. Some direction-finding and marine navigation systems such as the Russian Alpha transmitter use signals of constant frequency and amplitude, but such unmodulated VLF signals of significant amplitude have not been transmitted in Australia since the Omega navigation system ceased operating in 1997, made obsolete by global positioning systems (GPS).

For extracting geophysical information, assuming a constant frequency of military VLF transmissions has been a workable assumption, but for the purposes of removing the VLF signal, the assumption of constant frequency is inappropriate. There are many applications of radio communications and many methods of encoding information within broadcast signals (Sklar, 2001). Morse code is a very simple but slow method of communication that uses an interrupted signal to transfer information. Because of bandwidth and transmission efficiency considerations, high carrier-wave frequencies with modulation are needed to transmit and receive audio and video information (Sklar, 2001). These signals, with carrier frequencies in the range of $500 \mathrm{kHz}$ to $5 \mathrm{GHz}$ will not be considered in this paper, but they are used in engineering geophysics in the radio-magnetotelluric method (Oskooi and Pedersen, 2005; Tezkan and Saraev, 2008; Everett, 2013).

\section{METHODS}

\section{Observed very low frequency signals}

In a recent AMT experiment (Hennessy and Macnae, 2015), we collected streamed $B$ and $d B / d t$ data using an ARMIT sensor (Macnae, 2012; Macnae and Kratzer, 2013) near Heathcote, Victoria, at a sampling frequency of $120 \mathrm{kHz}$. I extracted a few seconds of $d B / d t$ data from this data set for VLF analysis.

Figure 3 plots the response of north-south and east-west components of the $d B / d t$ sensors for 5-ms duration. In this short time frame, three small amplitude sferics labeled S1, S2, and S3 can be seen, with $\mathrm{S} 1$ and $\mathrm{S} 2$ having stronger east-west signals, and S3 having a stronger north-south component. The north-south component has a larger background response between the sferics, which, from the timing of successive peaks, can be determined to be at a frequency of approximately $19.8 \mathrm{kHz}$. This is the VLF signal from station NWC. Apparent amplitude variations in the steady signal are mainly due to the noninteger ratio between the transmission and sampling frequencies.

To establish the frequencies and directions of all the VLF sources detectable in the streamed data, I used the MATLAB signal processing function pwelch (Mathworks, 2015) to estimate the spectral content of $100 \mathrm{~s}$ of east-west and north-south data using an overlapped segmentaveraging algorithm. Figure 4 plots these spectral amplitudes in the VLF frequency range with selective spectra enlarged in Figure 5.

Using directional estimation (predicted using the arctangent of the relative amplitudes of the north-south and east-west components, assuming cylindrical propagation with $d B / d t$ perpendicular to the direct path from the source), signals from five of the six transmitters plotted in Figure 1 were identified, with the four clearest being
VTX (southern India) at $16.3 \mathrm{kHz}, \mathrm{NWC}$ (Western Australia) at $19.8 \mathrm{kHz}$, JJI (Ebino, Japan) at $22.2 \mathrm{kHz}$, and a source, probably $3 \mathrm{SA}$ or $3 \mathrm{SB}$ in China at $24.1 \mathrm{kHz}$. Of immediate interest is that these detailed spectra (Figure 5) appear to show significant differences in the detailed character of the transmissions.

The Chinese transmitter (Figure 5d) has five distinct frequency spikes with 25-Hz separation, and the NWC (Figure 5b) and Japanese (Figure 5c) transmitters have a smooth spectral peak of 100-200 Hz wide with sidebands evident in the larger NWC signal. The Indian transmitter (Figure 5a) has a spectral "comb" lying asymmetrically above the center of a peak similar in amplitude and shape (Figure $5 \mathrm{c}$ ) to the Japanese transmitter.

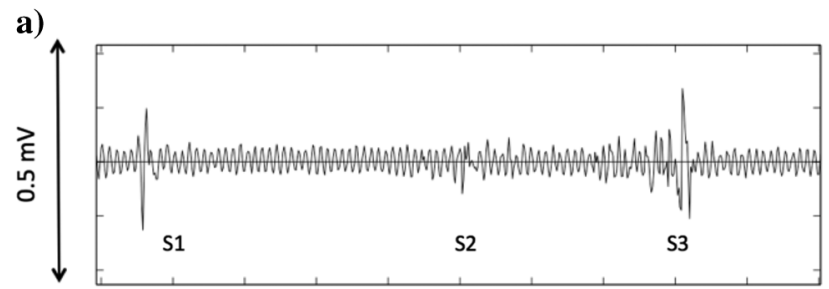

b)

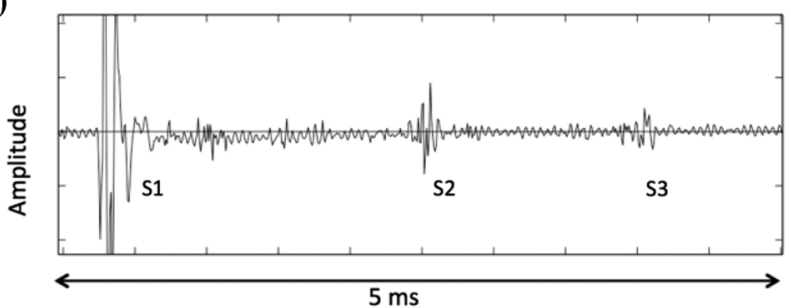

Figure 3. About $5 \mathrm{~ms}$ of $2 \mathrm{C} d B / d t$ data showing a steady VLF signal background that is larger in panel (a), the north-south direction, than in panel (b), the east-west component. Three small sferics, S1, S2, and S3, have amplitudes larger than the VLF signals and durations of less than $1 \mathrm{~ms}$.

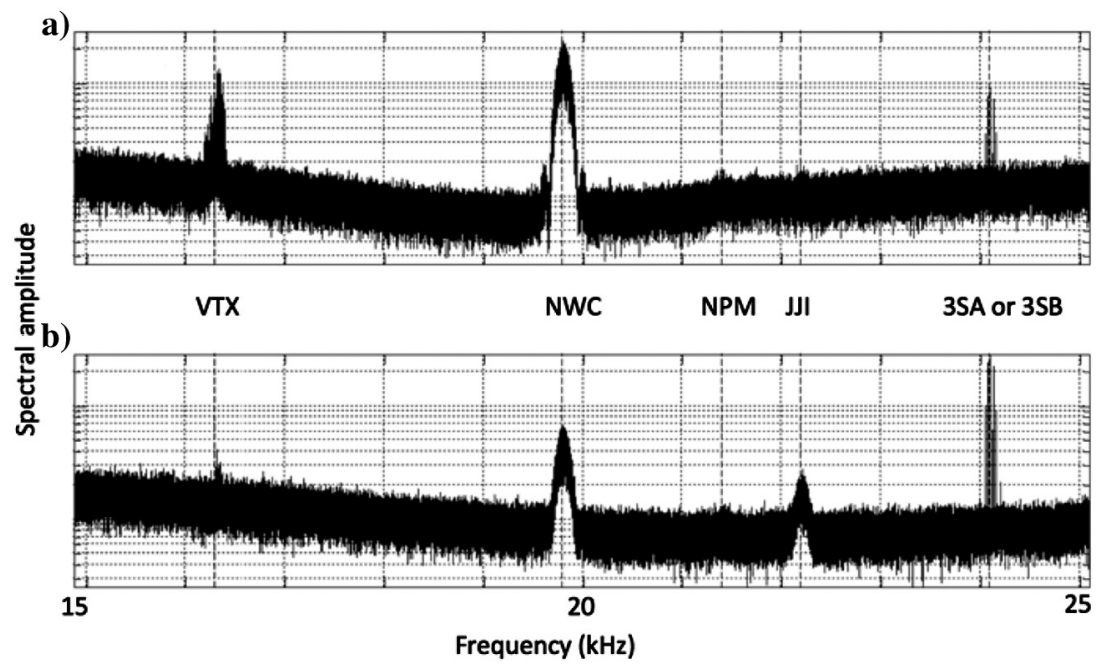

Figure 4. Observed VLF spectral amplitudes near Heathcote, Victoria. The largest signals come from Western Australian station NWC at $19.8 \mathrm{kHz}$. Station VTX (India) is at $16.3 \mathrm{kHz}$, and JJI (Japan) is at $22.2 \mathrm{kHz}$. Barely visible is station NPM (Hawaii) at $21.4 \mathrm{kHz}$. Signals at $24.1 \mathrm{kHz}$ originate from a transmitter believed to be in China. The relative amplitudes of the (a) north-south and (b) east-west components are consistent with the directions to sources as plotted in Figure 1. 


\section{Binary modulation}

Because of the high conductivity of seawater, VLF or lower frequencies are needed for any significant penetration to a submarine antenna. Even with high transmitter powers, signal levels are low, and as a result signal modulation or the bit rate is slow. No authoritative list of bit rates is available because these vary, and published bit rates are usually incorrect in my experience. Bit rates of known systems are asserted to be between 50 and 200 bits/s in web

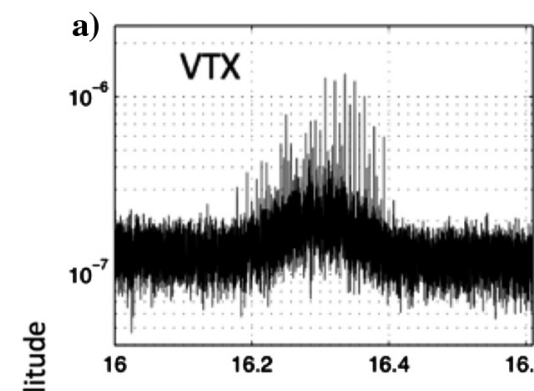

b)

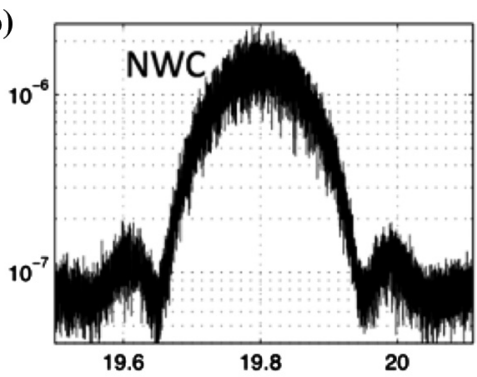

c)

ปัँّ

)

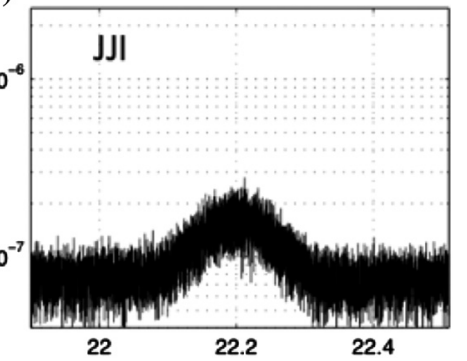

d)

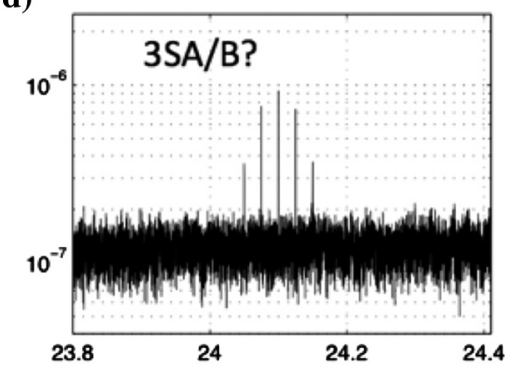

searches of unreliable websites. The most energetically efficient encoding system with constant peak amplitude and no phase discontinuities is the minimum shift keying (MSK) system used by United States military transmitters.

There are many alternative binary coding systems that have been used since the era of Morse code, and some of the methods relevant to VLF transmissions and this article are illustrated schematically in Figure 6. Generic methods of coding are amplitude shift keying (ASK), phase shift keying (PSK), and frequency shift keying (FSK). More than 1000 encoding schemes based on the three generic methods are classified in the radio communications literature (Industry Canada, 2012).

Figure 6 shows schematically two waveforms with ASK, specifically, the simple A1A on-off coding related to Morse code and the frequency-modulated A2A system, which allows modulation, usually at audio frequency, of a higher frequency carrier waveform. The PSK system shown must have at least one of an abrupt amplitude change or an abrupt slope change, with Figure 6 showing a slope change at the start of every change in bit. Three variants of the FSK system are shown including F1B, of which MSK is a special case, and a modulated F2B scheme.

The spectral amplitudes of the binary modulation schemes of Figure 6 are shown in Figure 7. Rather than the 2.75 and 3.25 cycles per bit coding seen in the schematic MSK in Fig 6, realistic MSK encoding of $200 \mathrm{bits} / \mathrm{s}$ with carrier $19.8 \mathrm{kHz}$ has [98.75, 99.25] cycles for [0, 1] bit coding, respectively. Taking the example of NWC, this transmitter has a bit rate of $200 \mathrm{~Hz}$ with a central frequency of $19.8 \mathrm{kHz}$. In fact, the transmitter uses two distinct frequencies 19,750 and $19,850 \mathrm{~Hz}$ with the offset of $50 \mathrm{~Hz}$ from the nominal $19.8 \mathrm{kHz}$ being exactly one-quarter of the bit rate. With a sine wave as the reference, the phase at the start of each bit can be any of $0^{\circ}, 90^{\circ}$, $180^{\circ}$, or $270^{\circ}$, chosen so as to ensure amplitude and phase continuity. These four phases can equally be considered as two waveforms, sine and cosine, both with zero phase but each having either a positive or negative sign.

Any or all of these encoding systems can be further modulated with a Gaussian window that reduces sideband amplitudes. This method is used, for example, in the Gaussian MSK encoding for mobile phones, but it will not be discussed here.

Submarines assuredly are able to extract binary sequences from VLF transmissions, of which sequences can be decrypted using secret methods. Conventionally, in a system with known digitization and check-sum or other redundancy built in, such as mobile phone communications, the Viterbi algorithm (Sklar, 2001) is used to extract a maximum likelihood bit sequence in the presence of noise. Unfortunately, the knowledge of permissible bit sequences in worldwide military VLF

Figure 6. ( $\mathrm{a}$ and $\mathrm{b}$ ) Some binary encoding systems of the bitstream 1011 include ASK with simple A1A and modulated (A2A) variants. PSK has slope and/or amplitude discontinuities associated with each bit change. FSK can use a different frequency for each bit (F1B) or different modulation frequency of a carrier (F2B). The most efficient form of FSK is MSK. Actual VLF transmissions contain many more cycles per bit than this schematic plot of waveforms. 
transmitters is not available to the field or processing geophysicist. A bit extraction method without encryption, redundancy, or other prior knowledge is required. Methodologies for extracting binary sequences can be analog or digital, and the Internet has several suggestions for use with home-built antennae, standard computer sound card digitization, and subsequent numerical analysis.

\section{RESULTS}

\section{Deriving the very low frequency bit sequence}

For this investigation, I used 120-kHz streamed data from an AMT sounding, collected near Heathcote, Victoria. For the 200 bits/s used in the NWC and NPM transmitters, each bit is $5 \mathrm{~ms}$ long. For NWC at a central frequency $f 0$ of $19.8 \mathrm{kHz}$, four correlation waveforms were created consisting of sine and cosine waves at $f 1=19,750 \mathrm{~Hz}$ and $f 2=19,850 \mathrm{~Hz}$. Each 5 -ms bit is 600 samples long at the sampling frequency. Initially, I convolved the two sine and two cosine correlation wavelets, each being one bit long with the data, and I attempted to locate the correlation amplitude peaks as the center of each bit. This worked well for the large NWC signal but poorly for all other VLF signals, in which the amplitude is closer to the noise level. One detection issue with MSK is that the two frequencies are not necessarily orthogonal when integrated over one bit. Although $\sin (f 1) \sin (f 2)$ and $\cos (f 1) \cos (f 2)$ are orthogonal, $\sin (f 1) \cos (f 2)$ and $\cos (f 1) \sin (f 2)$ are not. Prior to decoding and the establishment of a phase reference between the acquisition system and the VLF signal, it is not known whether the start of a bit has unit (cos) or zero (sin) amplitude. The signals are, however, exactly orthogonal when integrated over two successively identical bits. I therefore investigated the effect of convolving the data with sine and cosine correlation wavelets 2 bits long. Figure 8a plots the results of this correlation with two dashed lines (the rms amplitude at each frequency $f 1, f 2$ ) with the difference between them shown as a solid black line. The bit sequence starting 000101 is identified by symbols on the axis for the NWC transmitter. Two or more consecutive bits of the same value appear as a flat section of the correlation difference; one isolated bit appears as a single peak of about half the amplitude.

A 0.25 -ms sample (1/15 of a bit) of fitted data is shown in Figure 9. This short length was chosen so that the details of the fit were visible. Our object in this research was not, however, to extract bit sequences. Our aim is rather to subtract the VLF signal(s) from the observed data. To predict the VLF signal three things are needed: the exact frequency pair corresponding to bits zero and one, the amplitude, and the phase with respect to the local time base at the acquisition system.

If the data acquisition and transmitter systems were tied to the same clock, the phase (or more strictly the locally observed time of frequency change from $f 0$ to $f 1$ ) would only need to be es- timated once. In practice, Thomson et al. (2011) report "NWC, NPM, and NLK, like other U.S. Navy VLF transmitters, typically have very good phase and amplitude stability. However, as with the other U.S. transmitters, they normally go off-air once a week for 6 $8 \mathrm{~h}$ for maintenance. On return to air, the phase is still normally stable but the value of the phase (relative to GPS or UTC) is often not preserved. In addition, in the course of a typical week, there may be some gradual phase drift or a small number of additional times when there are random phase jumps." With geophysical data tied to GPS, processing must therefore adapt to phase drifts.

There are 600 samples in one bit of the NWC waveform in the sampled data. In Figure 8a, the heavy black difference can be seen to cross zero 15 times. The location of these zero crossings is spaced an integer number $n$ of bits ( $600 n$ samples) apart. Figure 10 shows a histogram of the positive remainders after $m * 600$ is subtracted from the data, where $m$ is the largest integer leaving a positive remainder. This plot of the variations in the timing of zero crossings has a median offset of 449 samples (Figure 10). This median was chosen as the local zero phase reference for this $1 \mathrm{~s}$ of data, the relative time of $(449+m * 600) / 120,000$ being the time of the end of the $m$ th bit, and the start of the $(m+1)$ th bit. Figure 11 presents the fitted signal amplitude for each presumed a)

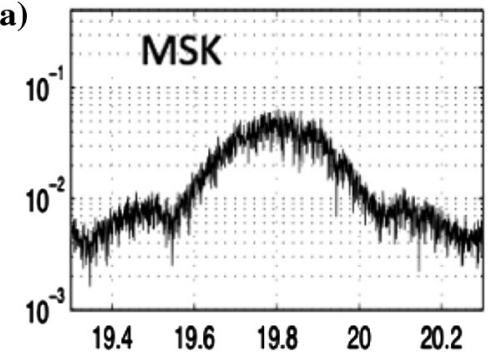

c)

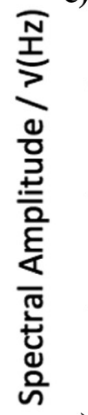

e)

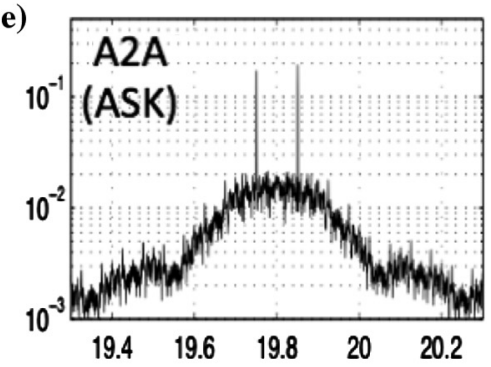

b)

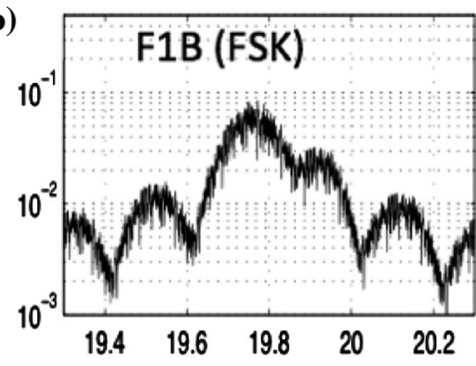

d)

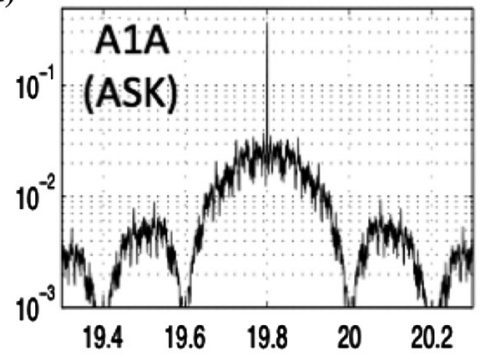

f)

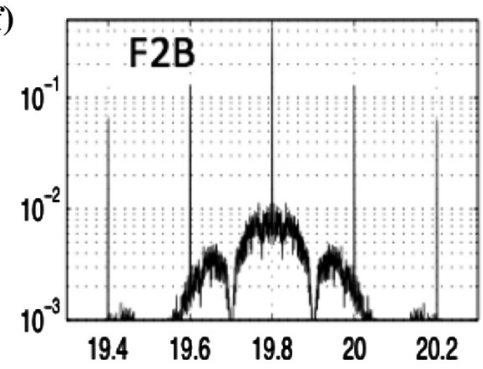

Frequency $(\mathrm{kHz})$

Figure 7. Using a central frequency of $19.8 \mathrm{kHz}$ and a bit rate of $200 \mathrm{~Hz}$, this is a plot of spectral amplitude versus frequency for $8 \mathrm{~s}$ of random binary data encoded with (a) MSK, (b) FSK, (c) PSK, (d) ASK, and (e and f) the modulated versions of ASK and FSK. 
bit. The north-south and east-west components have consistent fitted amplitudes with variations generally associated with the presence of large sferics evident in the raw data. To minimize these amplitude "errors," raw data were clipped to roughly double the average VLF signal amplitude before fitting on a bit-by-bit basis. The assumed amplitude for later stripping is taken as the median amplitude of the fitted values.

It is worth noting that MSK encoding permits the recovery of a missing or incorrect bit. This is because if the phase of the previous bit and the subsequent bit is known, there is a unique choice of frequency for the missing binary digit. With reference to Figure 6, it can be seen that during a 1 bit, the phase rotates $90^{\circ}$, whereas during a 0 bit, the phase rotates $180^{\circ}$. If it were possible to sample the noise-free amplitude or phase at exactly every transition, the MSK bit sequence could be extracted. Consistency checks are very useful if large sferics lead to an error or ambiguity in any isolated fit to a bit. Intended receivers of MSK data are likely to have information on encoding and data redundancy that can further improve signal extraction with the Viterbi algorithm.

\section{Stripping the minimum shift keying prediction}

Once an amplitude, bit timing, and frequency are defined for each bit, the MSK encoding scheme that ensures amplitude and phase continuity are used to generate a waveform using segments of sine and cosine waves. This can then be subtracted from the observed data. Figure 12a shows the short segment of north-south data seen
Figure 8. (a-e) Results (dashed lines) of correlating the observed time series with a VLF wave of the duration of 2 bits at MSK frequencies $f 1$ and $f 2$ on either side of the central or nominal frequency $f 0$. The heavy black profiles are the difference between the two fitted curves. The diamonds plotted on the zero axes indicate the timing of the center of each bit, with the black marker when the difference curve is positive indicating one and an open marker zero when the difference curve is negative.

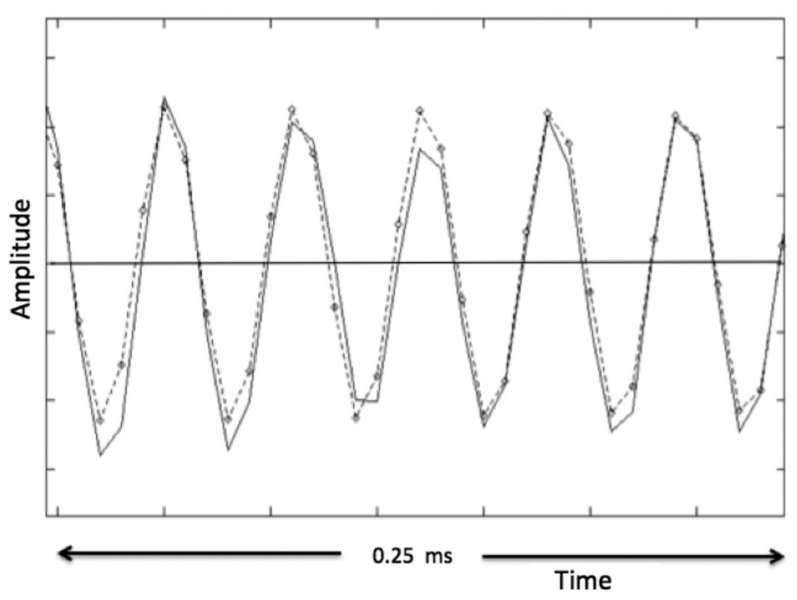

Figure 9. Fit of MSK showing data (solid) and fit (symbols) over approximately 6 VLF cycles taking $0.25 \mathrm{~s}$. The amplitude fit is by least-squares over one bit $(5 \mathrm{~ms})$. a)

b)

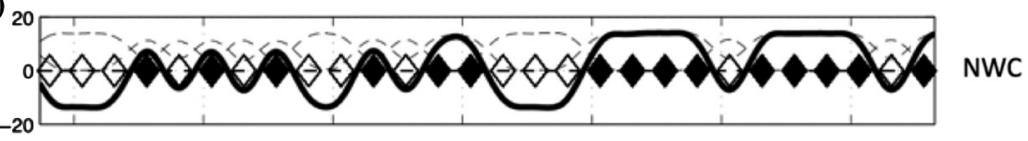

)

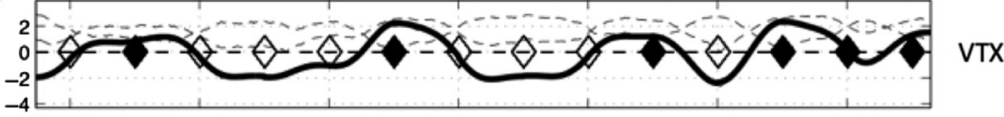

응)

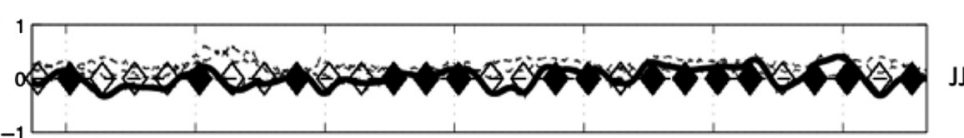

d)

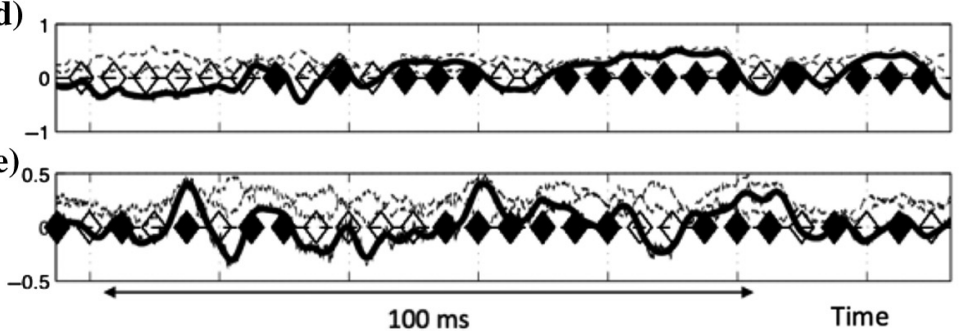

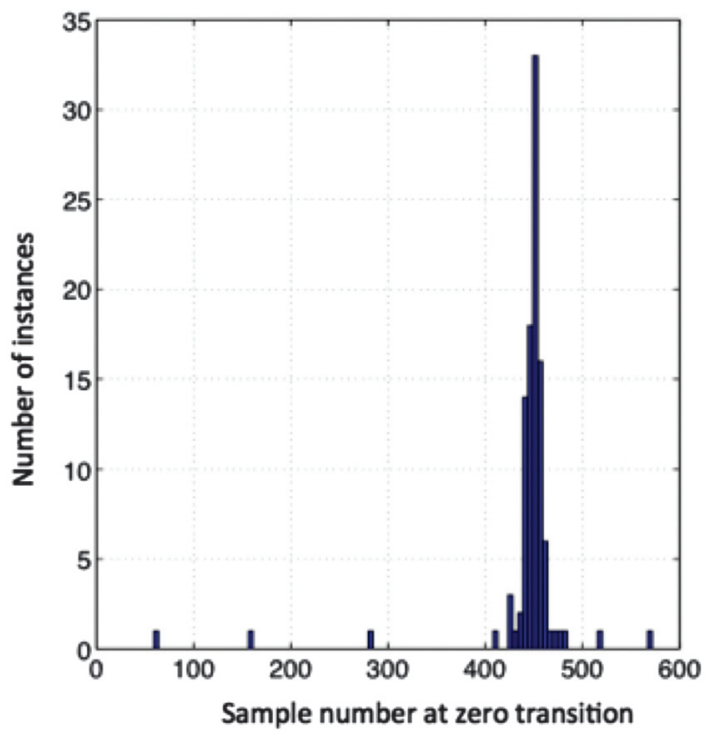

Figure 10. Histogram of the sample offset (phase) zero crossings for the NWC bit detector. One bit is 600 samples long. 
in Figure 3. Between 113 and $114 \mathrm{~ms}$, there are no obvious sferics. The normalized rms signal in this $1 \mathrm{~ms}$ of data is $16.8 \mathrm{ppm}$. Figure $12 \mathrm{~b}$ shows the time series after the predicted NWC signal has been subtracted. In the regions between sferics, the amplitude is much reduced with the rms value between 113 and 114 ms less than one third of what is was before.

After the automated algorithm stripped the predicted NWC signal, the process was repeated successively for the different trans-
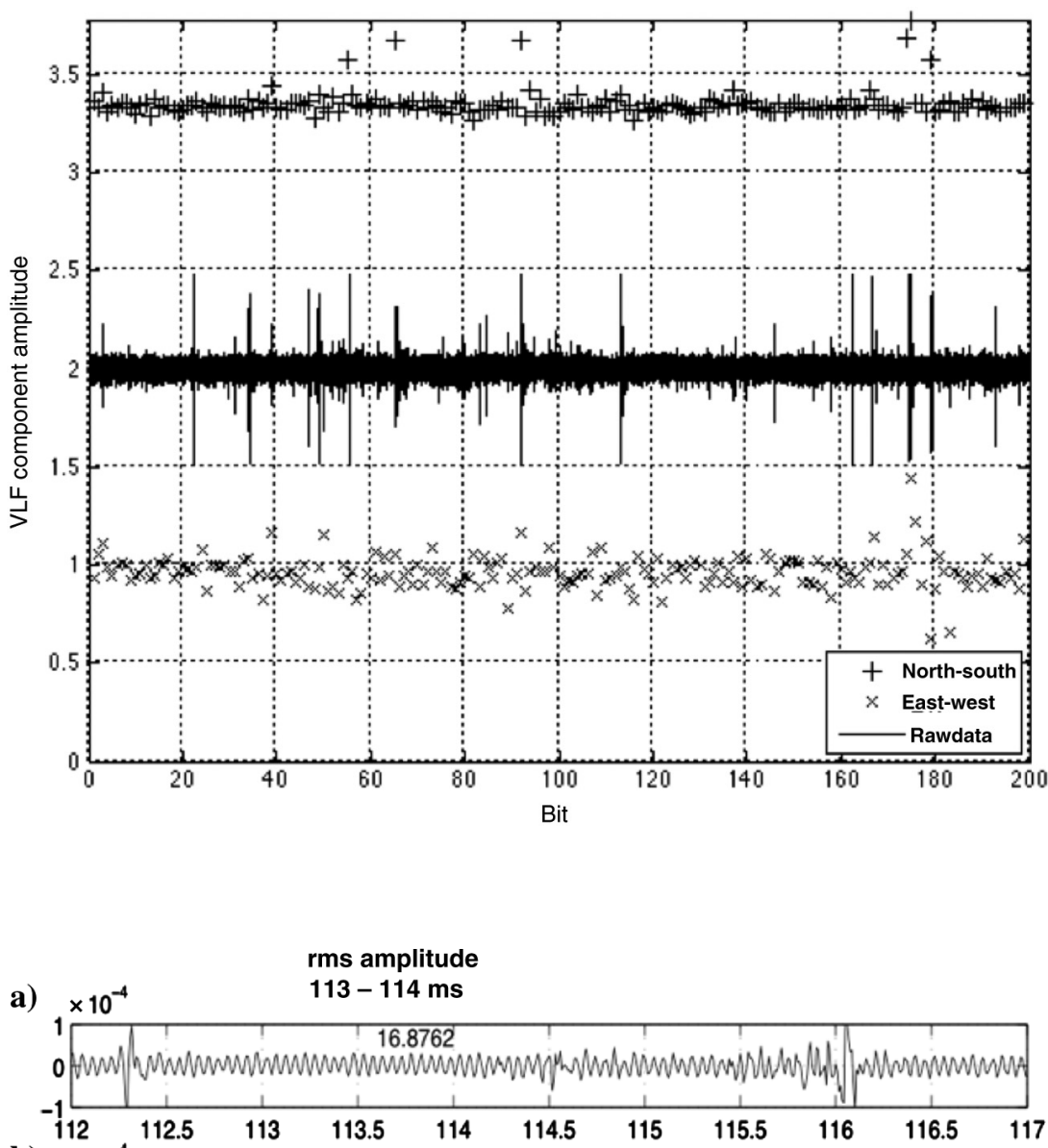

b)

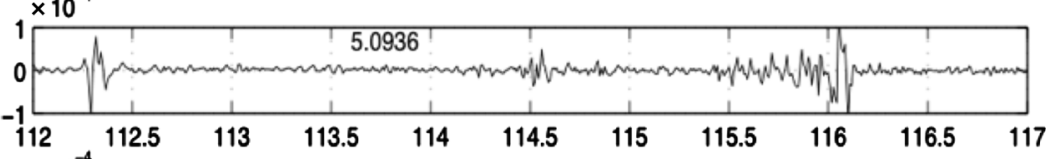

c) $\times 10^{-4}$

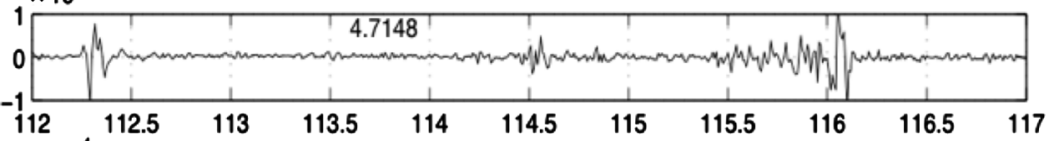

d)

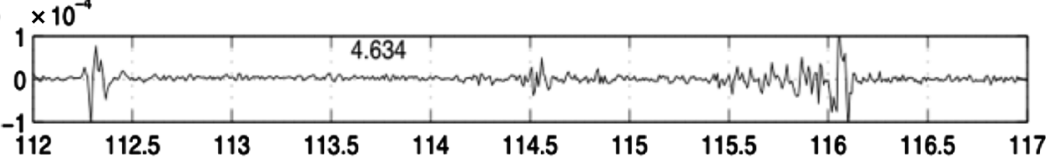

e)

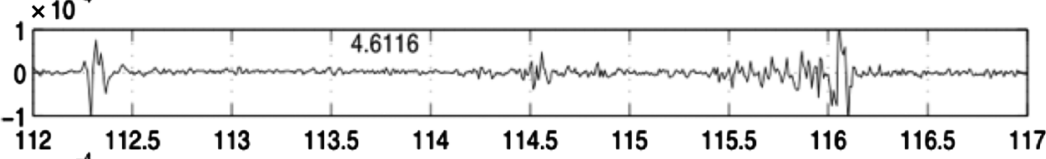

f)

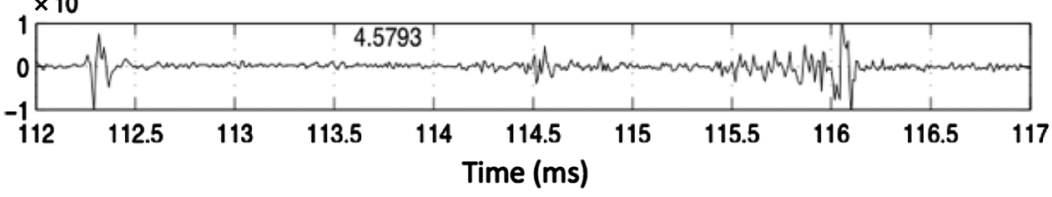

Figure 11. Fitted amplitude of individual bit signals over a $1 \mathrm{~s}$ duration for both components of the NWC signal. Variations in the fitted amplitude (symbols) are generally associated with the presence of sferic spikes on the streamed data. The overall amplitude stability is evidence of a constant source amplitude, and the component stability additionally is evidence of a consistent transmission path

Figure 12. Progressive stripping of a short segment of the observed time series: (a) original data, (b) NWC stripped, (c) NWC + VXT stripped, (d) + JJI stripped, (e) + NPM stripped, and (f) + 3SA/B? stripped. The rms amplitude of the residual signal in a segment of data between sferics (113-114 ms) decreases as each VLF signal is predicted and stripped. 
mitters detected. The second signal to be decoded was the VTZ transmission. The MSK decoding at $200 \mathrm{bits} / \mathrm{s}$ with frequencies $f 1, f 2$ displaced by $50 \mathrm{~Hz}$ from the $16.3 \mathrm{-kHz}$ center was unsuccessful. However, decoding with $100-\mathrm{Hz}$ offset frequencies and 100 bits/s produced the result seen in Figure 8b. With this smaller signal, there was much less reduction in the residual rms after subtraction of the modeled transmission. The automated MSK stripping algorithm was then applied to other detected transmissions. Each successive fit and strip reduced the residual rms in our 1-ms sample between sferics (Figure 12). The spectral amplitude of the observed signal before and after each successive strip is shown in Figure 13.

An issue not addressed in this paper is the modeling of transmitted signals from different encoding systems. The Chinese transmitter (presumed to be 3SA or 3SB) has spectral characteristics completely inconsistent with MSK (Figures 5 and 6), and in the $1 \mathrm{~s}$ of data discussed in this article, the fitted MSK bits shown in Figure 8e do not look trustworthy, and, further, the stripping has not reduced the power in the $24.1-\mathrm{kHz}$ region (Figure 13).
Even if military VLF communications can be decoded and exactly modeled and stripped, it cannot be expected that this stripping would reduce the residual VLF signals to zero. There are several sources of natural VLF signals including lightning and magnetospheric currents (Barr et al., 2000; Reuveni et al., 2011), and many cultural sources such as switching power supplies and other electronics used in data acquisition systems and computers.

The larger an MSK signal is, the more reliable decoding and hence modeling and subtraction will be. The stripping method described should therefore, if anything, work better with larger VLF signals because there will be a lower chance of incorrect bit prediction. This is illustrated with a data set collected in Western Australia, at test site 2 located approximately $1100 \mathrm{~km}$ south of the NWC transmitter, one-third of the 3300-km distance from NWC to Heathcote (test site 1, Figure 1). The spectrum of the data is presented in Figure 14. This large signal was fitted and stripped by the methods described above, and the result is shown in Figure 15. A
Figure 13. Spectral amplitude versus frequency from $1 \mathrm{~s}$ of north-south $d B / d t$ data (a) before and (b) after stripping of the VLF signals using an MSK encoding assumption.
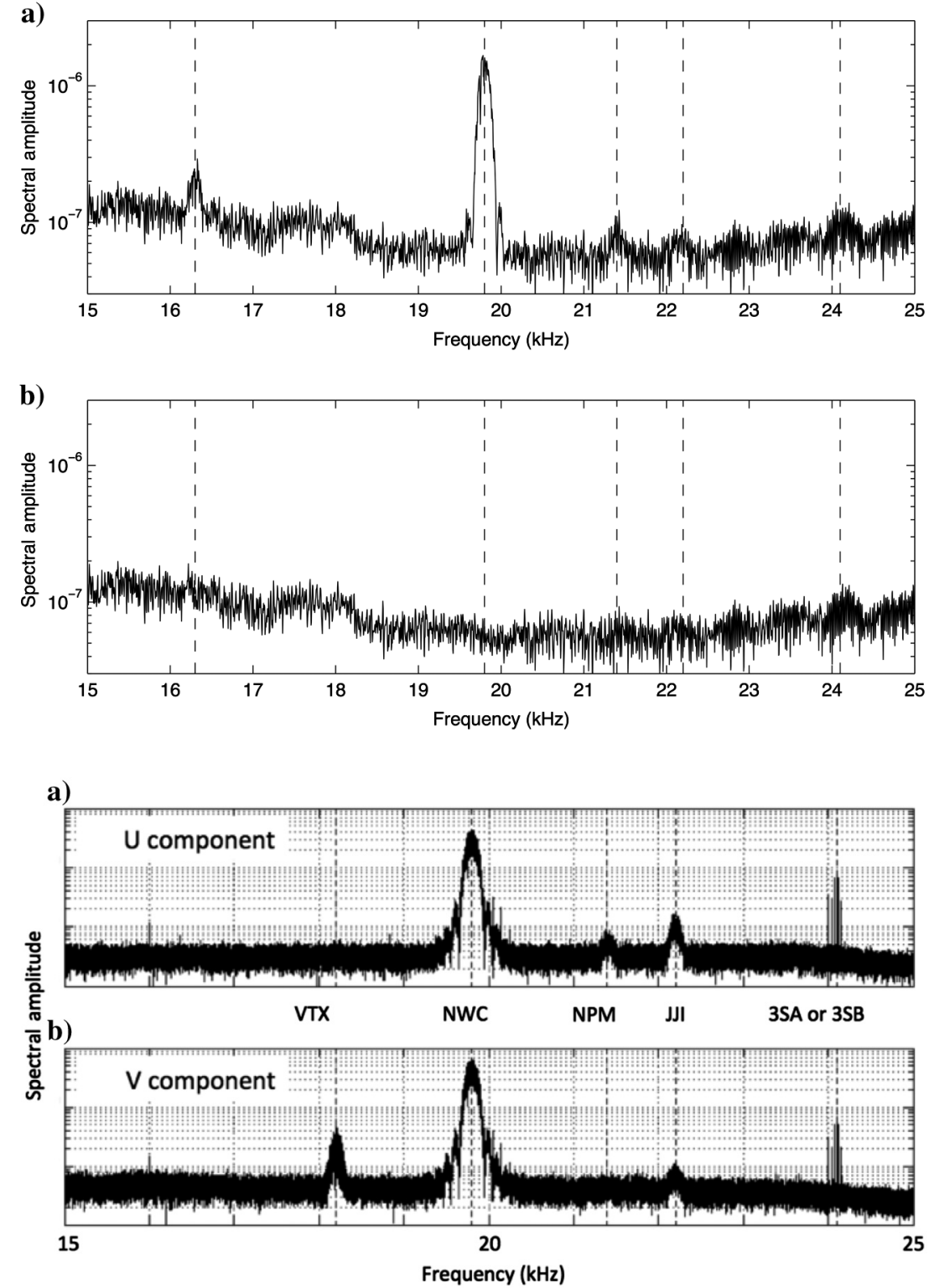

Figure 14. (a) U component and (b) V component. Spectral amplitudes observed at test site 2 in Western Australia, approximately $1100 \mathrm{~km}$ south of NWC. The Indian VTX station was broadcasting at $18.2 \mathrm{kHz}$ when these data were collected in 2013. The two orthogonal horizontal axes are labeled $\mathrm{U}$ and $\mathrm{V}$, and as the sensor was airborne, their orientations were rotated from the northsouth and east-west directions. The sensor here had a different gain from that used at site 1 , and the data were sampled at $50 \mathrm{kHz}$ rather than $120 \mathrm{kHz}$ of the previous example. 
a)

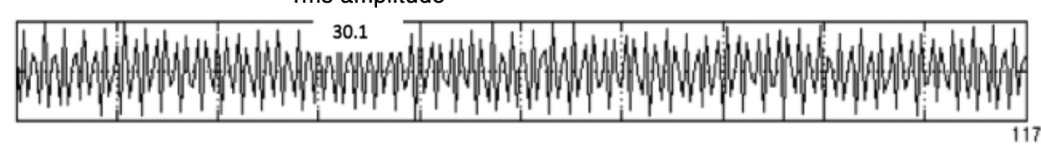

b)

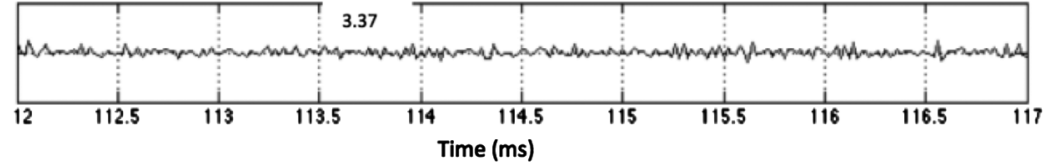

Figure 15. ( $a$ and $b$ ) Time series of $5 \mathrm{~ms}$ duration to show the proportionately greater reduction in unwanted VLF signals for test site 2 than was the case with smaller VLF amplitudes at test site 1 (Figure 12).

factor of nine reduction in the rms noise in a 1-ms test range without large sferics was achieved with the MSK stripping algorithm.

\section{CONCLUSIONS}

The frequency-domain approach of notch filtering or bandwidth limitation is unsatisfactory when early delay-time data are required, or for the analysis of sferic signals in audio frequency magnetotellurics. United States military VLF transmitters presently communicate $200 \mathrm{bits} / \mathrm{s}$ and are MSK encoded, and it is possible to derive the encrypted bitstream from data that have been sampled at typical geophysical data streaming rates. The transmitted signal can then be exactly and automatically recreated, and the predicted signal at the receiver can be subtracted from the data stream. It is possible to predict bit rates and encoding methods from other military transmitters through data analysis, and once the corner frequency and bit rate is determined, it is possible to automatically subtract the predicted communication signals from streamed data. Future work will apply this stripping methodology to determine the improvement actually achieved on early time-domain EM data. If the VLF data stream is treated as unwanted signal or noise in a controlled-source EM measurement, the factor of three to nine improvement achieved in eliminating unwanted signals should provide a major improvement in survey signal/noise. In particular, I predict that the stripping methodology will prove of major benefit to ground or AEM surveys within a few hundred kilometers of a VLF transmitter.

\section{ACKNOWLEDGMENTS}

I thank J. Hamad and L. Hennessy for field assistance in the Victorian AMT data acquisition. The Western Australian data came from an airborne survey collected during AMIRA International Project P1136a. R. Smith, B. Oskooi, and an anonymous reviewer are thanked for their constructive comments that significantly improved the paper.

\section{APPENDIX A}

\section{NOTCH FILTERS APPLIED TO MINIMUM SHIFT KEYING SIGNALS}

At least one assertion in the literature is that notch filters can be used to eliminate VLF signal noise in AEM systems. This statement is contrary to my experience that only high-cut filters are useful, but that these affect data for the first few tens of microseconds after controlled-source waveform transitions. Analog filters as used in geophysical instrumentation can be mathematically represented as infinite impulse response filters. I analyze in this appendix the effects of such filters in reducing an MSK-encoded signal using the NWC specifications of 200 bits/s, with frequencies of 19,750 and $19,850 \mathrm{~Hz}$. Figure A-1 shows in black the spectral amplitude of $8 \mathrm{~s}$ of an MSK waveform derived from a random bitstream. Even though the raw data contain only 19,750 and $19,850 \mathrm{~Hz}$ frequencies in 5-ms segments, the overall spectrum as numerically estimated in MATLAB by pwelch (Mathworks, 2015) is broad. With the central amplitude peak in the spectrum of $200-300 \mathrm{~Hz}$ wide, I first designed and applied a 200-Hz-wide notch filter using MATLAB routine iirnotch. I then filtered the 8-s of synthetic data, and the resulting spectral amplitude is also shown in Figure (A-1) in gray. This filter reduces the central peak by less than an order of magnitude, and it has a minimal effect on the sideband amplitudes.

Because the MSK transmission from NWC actually contains only 19,750 and $19,850 \mathrm{~Hz}$ frequencies in 5-ms segments, it is instructive to see the effect of applying narrowband filters at these two frequencies. The result is shown in Figure A-2 for a 1-Hz-wide notch. Almost no reduction has been made to the overall spectrum. The reason for this is the inability of an infinite impulse response notch filter to reduce signals with significant phase changes over nonadjacent bits. Figure A-3 shows the effect of applying two 50-Hz-wide notch filters with centers at the same two frequencies to the MSK-encoded transmission. The overall result is better than the 1-Hz-wide notches, but the overall spectral reduction limited.

The spectra of Figure 5 in the main text only showed two adjacent sidebands for the NWC transmission. Figure A-4 shows the spec-

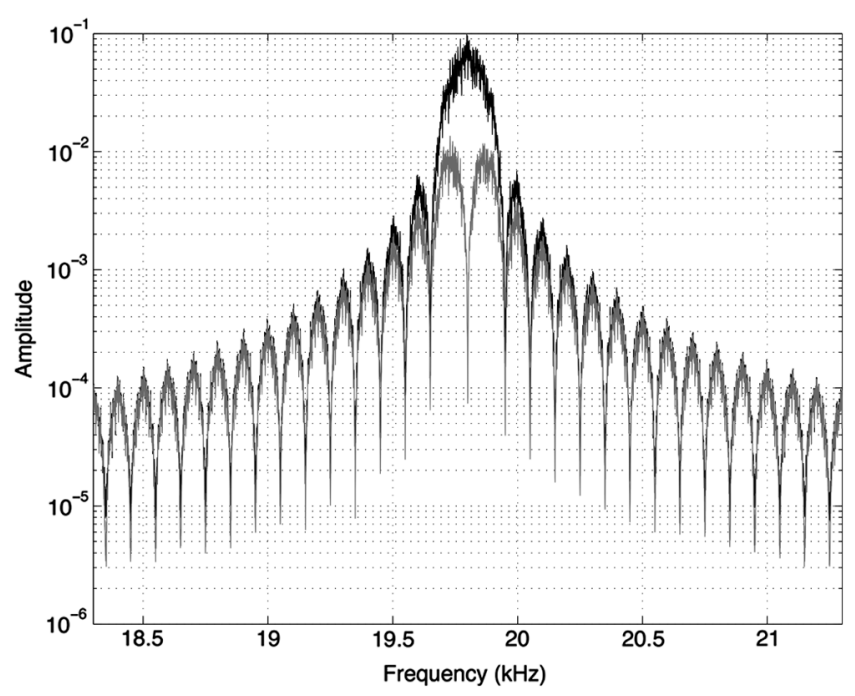

Figure A-1. An MSK spectral amplitude for $200 \mathrm{bit} / \mathrm{s}$ encoding and a central frequency of $19.8 \mathrm{kHz}$ (black), with the spectrum after a $200-\mathrm{Hz}$-wide notch filter was applied (gray). Sideband separation is $100 \mathrm{~Hz}$ apart from the central peak. 
trum for the case plotted in Figure A-1 with 5\% Gaussian noise added to the MSK-encoded waveform. In this case, the unfiltered spectrum (black) does show the two adjacent sidebands above noise, with the more distant sidebands more difficult to identify in the noise. The notch-filtered spectrum (gray) shows two peaks of lower amplitude than the original unfiltered main peak. The 5\% added noise hides all sidebands seen in Figure A-1, and it is similar to the Japanese JJI spectrum seen in Figure 5.

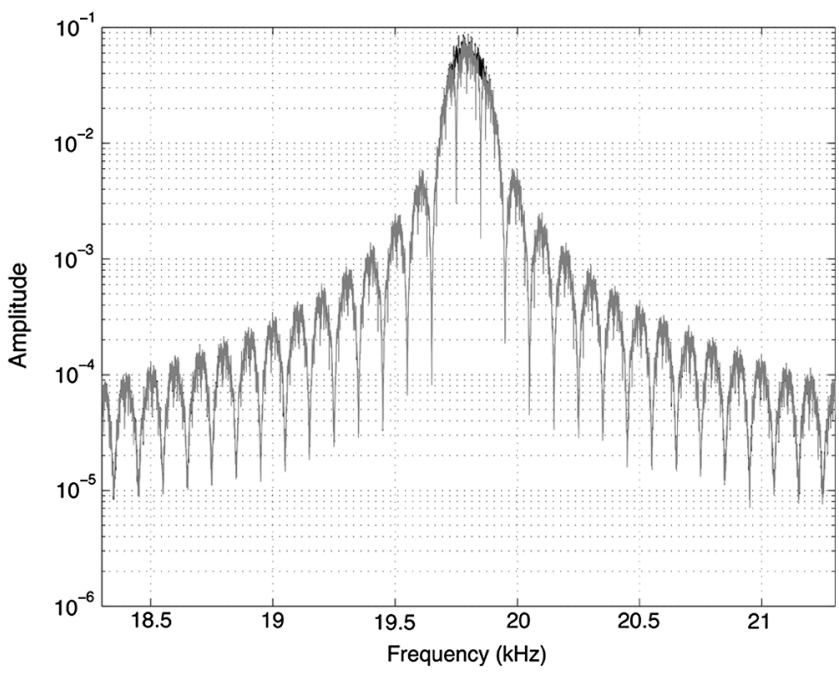

Figure A-2. The almost undetectable effect of filtering the MSK signal (black) with two successive narrowband filters at 19,750 and $19,850 \mathrm{~Hz}$ (gray).

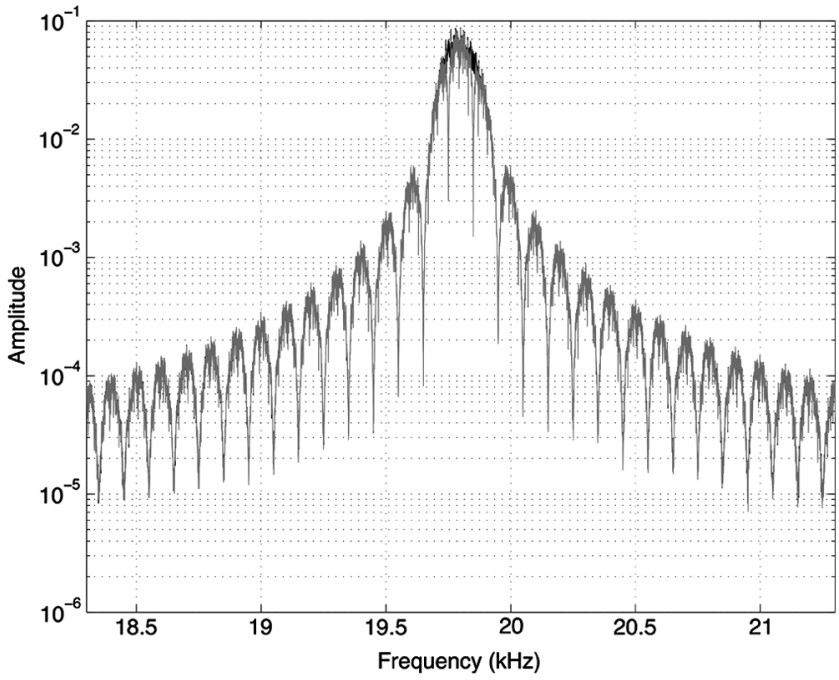

Figure A-3. Effect of successive filtering with $50-\mathrm{Hz}$-wide filters at 19,750 and $19,850 \mathrm{~Hz}$. Some reduction in the peak amplitude is now achieved, with no reduction in the sideband amplitudes.

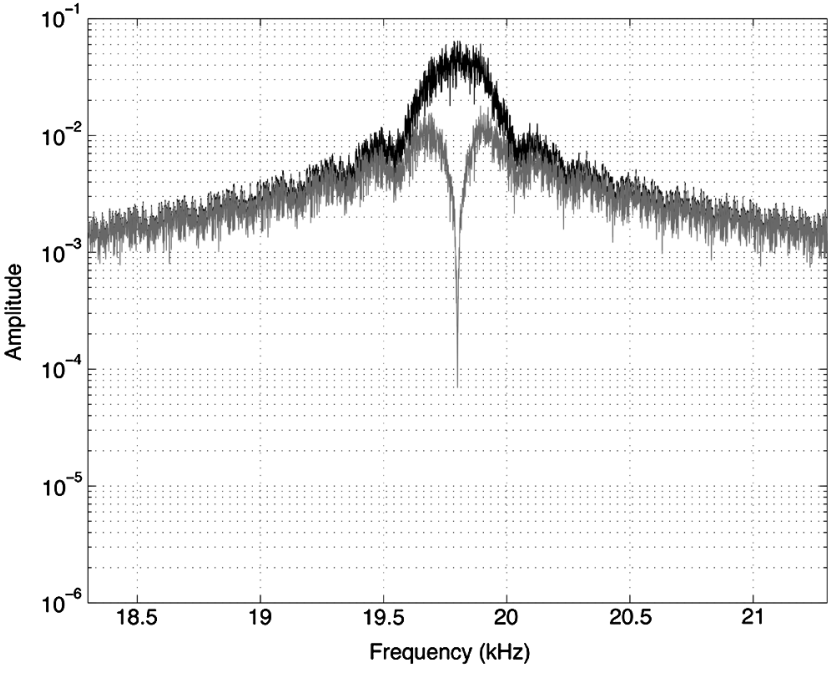

Figure A-4. The spectral amplitude (black) when 5\% random noise is added to the MSK signal and after filtering with a $200-\mathrm{Hz}$-wide, $19.8-\mathrm{kHz}$ filter (gray).

\section{REFERENCES}

Allard, M., 2007, On the origin of the HTEM species, in B. Milkereit, ed., Proceedings of Exploration 07: Fifth Decennial International Conference on Mineral Exploration, Decennial Mineral Exploration Conferences, 355-374.

Barr, R., D. Llanwyn Jones, and C. Rodger, 2000, ELF and VLF radio waves: Journal of Atmospheric and Solar-Terrestrial Physics, 62, 1689-1718, doi: 10.1016/S1364-6826(00)00121-8.

Christiansen, A., E. Auken, and A. Viezzoli, 2011, Quantification of modelling errors in airborne TEM caused by inaccurate system description: Geophysics, 76, no. 1, F43-F52, doi: 10.1190/1.3511354.

Efferso, F., E. Auken, and K. Sorensen, 1999, Inversion of band-limited TEM responses: Geophysical Prospecting, 47, 551-564, doi: 10.1046/j $.1365-2478.1999 .00135 . x$.

Everett, M., 2013, Near-surface applied geophysics: Cambridge University Press.

Hennessy, L., and J. Macnae, 2015, Natural field electromagnetics using a partially known source: Improvements to signal to noise ratios: Presented at 24th International Geophysical Conference and Exhibition, ASEG, Extended Abstracts.

Industry Canada, 2012, Designation of emissions, class of station and nature of service, Government of Canada TRC-43 Issue 3.

Lane, R., C. Plunkett, A. Prica, A. Green, and Y. Hu, 1998, Streamed dataA source of insight and improvement for time domain airborne EM: Exploration Geophysics, 29, 16-23, doi: 10.1071/EG998016.

Macnae, J., 2012, Design and testing of ARMIT magnetic field sensors for EM systems: Presented at 22nd International Geophysical Conference and Exhibition, ASEG, Extended Abstracts.

Macnae, J., 2015, Correcting EM system bandwidth limitations: Presented at 24th International Geophysical Conference and Exhibition, ASEG, Extended Abstracts.

Macnae, J., and T. Kratzer, 2013, Joint sensing of B and dB/dt responses: Presented at 23rd International Geophysical Conference and Exhibition, ASEG, Extended Abstracts.

Mathworks, 2015, Function documentation, http://it.mathworks.com/help/ signal/ref/pwelch.html, accessed 20 September 2015.

McNeill, J., and V. Labson, 1991, Geological mapping using VLF radio fields, in M. Nabighian, ed., Electromagnetic methods in applied geophysics: Part B, SEG, 521-640.

Oskooi, B., and L. Pedersen, 2005, Comparison between VLF and RMT methods. A combined tool for mapping conductivity changes in the sedimentary cover: Journal of Applied Geophysics, 57, 227-241, doi: 10 .1016/j.jappgeo.2005.01.002.

Oskooi, B., and L. Pedersen, 2006, Resolution of airborne VLF data: Journal of Applied Physics, 58, 158-175, doi: 10.1016/j.jappgeo.2005.06.002.

Pedersen, L., 1998, Tensor VLF measurements: Our first experiences: Exploration Geophysics, 29, 52-57, doi: 10.1071/EG998052.

Pedersen, L., and B. Oskooi, 2004, Airborne VLF measurements and variations in ground conductivity: A tutorial: Surveys in Geophysics, 25 151-181, doi: 10.1023/B:GEOP.0000028161.90659.4b. 
Reuveni, Y, C. Price, E. Greenberg and A. Shuval, 2011, Natural ELF/VLF atmospheric noise statistics in the eastern Mediterranean: Presented at General Assembly and Scientific Symposium, XXXth URSI, 1-3.

Samanes, J., J. P. Raulin, E. Macotela, and W. Guevara Day, 2015, Estimating the VLF modal interference distance using the South America VLF Network (SAVNET): Radio Science, 50, 122-129, doi: 10.1002/ 2014RS005582.

Schamper, C., E. Auken, and K. Sorensen, 2014, Coil response inversion for very early time modelling of helicopter-borne time-domain electromagnetic data and mapping of near-surface geological layers: Geophysical Prospecting, 62, 658-674, doi: 10.1111/1365-2478.12104.

Sklar, B., 2001, Digital communications: Fundamentals and applications 2nd ed.: Prentice Hall.

Tezkan, B., and A. Saraev, 2008, A new broadband radiomagnetotelluric instrument: Applications to near surface investigations: Near Surface Geophysics, 6, 245-252, doi: 10.3997/1873-0604.2008019.
Thomson, N., C. Rodger, and M. Clilverd, 2011, Daytime D-region parameters from long path VLF phase and amplitude: Journal of Geophysical Research, Space Physics, 116, A11305, doi: 10.1029/ 2011JA016910.

Vallée, M., M. Chouteau, and G. Palacky, 1992a, Effect of temporal and spatial variations of the primary field signal on VLF total-field surveys: Geophysics, 57, 97-105, doi: 10.1190/1.1443192.

Vallée, M., M. Chouteau, and G. Palacky, 1992b, Variations of the VLF-EM primary field: Analysis of airborne survey data, New Brunswick, Canada: Geophysics, 57, 181-186, doi: 10.1190/1.1443182.

Waheed-uz-Zaman, M., and M. Yousufzai, 2010, Study of the effects of radio waves propagation under sea at Pakistan coastal zones: Journal of American Science, 6, 413-419. 